\title{
Optimization Model of Cross-Border E-commerce Payment Security by Blockchain Finance
}

\author{
Tung-Chun Chen $\mathbb{D}^{1,2}$ Yu-Shen Liang, ${ }^{1}$ Po-Sheng Ko $\mathbb{D}^{3},{ }^{3}$ and Jui-Chan Huang $\mathbb{D}^{4}$ \\ ${ }^{1}$ Department of Plant Industry, National Pingtung University of Science and Technology, Pingtung 91201, Taiwan \\ ${ }^{2}$ Department of International Business, National Kaohsiung University of Science and Technology, Kaohsiung 82444, Taiwan \\ ${ }^{3}$ Department of Public Finance and Taxation, National Kaohsiung University of Science and Technology, Kaohsiung 82444, Taiwan \\ ${ }^{4}$ Yango University, Fuzhou, Fujian 350015, China
}

Correspondence should be addressed to Po-Sheng Ko; e333ee@nkust.edu.tw

Received 14 October 2021; Revised 29 November 2021; Accepted 2 December 2021; Published 24 December 2021

Academic Editor: Deepak Kumar Jain

Copyright (c) 2021 Tung-Chun Chen et al. This is an open access article distributed under the Creative Commons Attribution License, which permits unrestricted use, distribution, and reproduction in any medium, provided the original work is properly cited.

\begin{abstract}
Cross-border trade is also changing and innovating. Electronic payment has become the core application of modern cross-border e-commerce. However, although electronic payment has brought convenience and efficiency to enterprises and social life, there are also many problems in transaction information security. These problems not only hinder the development of electronic payment but also bring hidden dangers to people's property security. Therefore, an encryption algorithm of e-commerce was proposed, and its algorithm security and risk control mode was further studied by means of case analysis, empirical analysis, and comparative analysis. Experimental data show that blockchain technology is a breakthrough. The aim of the study is to explore its application in specific circumstances and strive to make the new e-payment mode in line with international standards.
\end{abstract}

\section{Introduction}

At present, e-commerce has been successfully integrated with many real economy, which is also the most effective transformation direction of the existing traditional business model. With the gradual opening of the concept of market consumption, catering, clothing, and various service industries have made new breakthroughs in the new ecommerce mode. It has changed from the earliest semimanual payment represented by postal bank transfer to the form of online banking separated from manual mode and then to the form of electronic wallet represented by Alipay. Online payment of user experience is developing and improving. At present, e-wallet is undertaking more and more functions. Online investment, online bill making, AA payment, and even online lending are emerging in an endless stream. It can be imagined that online payment tool will soon develop into a rich personal finance online service system $[1,2]$. Cross-border e-commerce refers to an international business activity in which transaction subjects belonging to different custom territories reach transactions through an e-commerce platform, conduct e-payment and settlement, and deliver goods through cross-border e-commerce logistics and remote storage, so as to complete the transaction. Cross-border e-commerce is developed based on the network. The network is a media body without boundary, which has the characteristics of globalization and decentralization. Therefore, the cross-border e-commerce attached to the network has the characteristics of globalization and decentralization.

Chen and Wang discussed c-commerce [3]. Here, we construct the existing five-fold classification of e-commerce to adapt to various e-commerce and e-commerce perspectives. Thus, Holsapple and Singh synthesize a complete overall definition of e-commerce. Its definition is to use electronic technology and data processing technology to solve information processing problems in the field of commerce and trade. It reflects the change of an enterprise production process, including resource allocation, production technology innovation, and management virtualization. In 


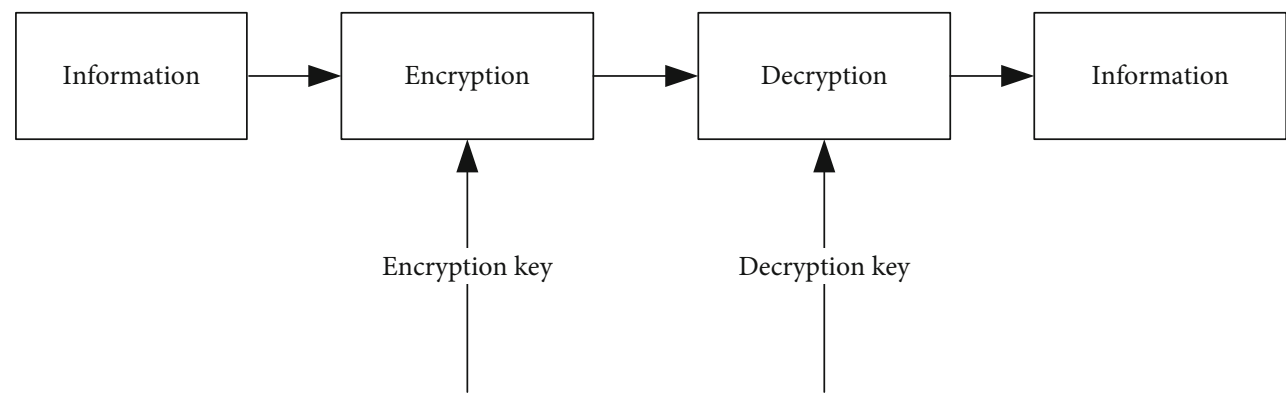

FIgURE 1: Information encryption flow chart.

order to overcome this problem, Holsapple and Singh put forward a knowledge management viewpoint; the knowledge management is the process of transforming the information obtained from various sources into knowledge and connecting with people, so as to formally manage knowledge. It was clearly recognized that e-commerce is based on electronic knowledge: the process and technology of managing knowledge. Holsapple and Singh argue that the knowledgeoriented e-commerce perspective is beneficial, providing knowledge-based e-commerce in e-organizations [4].

The blockchain finance technology takes literature analysis as the main method and empirical analysis and comparative analysis as the auxiliary method to establish an analysis model, and the optimization mode of cross-border ecommerce payment security by blockchain finance was studied.

\section{Research Methods of the Optimization Model}

2.1. E-commerce Payment Security Encryption Algorithm. (1) Customer privacy is a necessary condition for blockchain to gain public attention and rapid development [5, 6]. Many encryption technologies are used in blockchain, such as Merkle hash tree, digital signature, and ECC elliptic curve encryption. In essence, encryption technology is a technology that converts the original pure text information into ciphertext information that cannot be understood by some measures and then restores the original pure text information after decryption [7]. The process is shown in Figure 1. The main process is that the sender encrypts the information with an encryption secret key, then transmits it to the receiver, and then restores the information with the decryption key.

A Vigenere receiver is a famous letter. Key and plaintext are converted into corresponding numeric characters 1-92, so the ciphertext space is 92 .

Use key to encrypt information:

$$
K_{e}=Q E\left(F_{e}\right)=\left(F_{e}+N_{e}\right) \bmod \{92\} .
$$

Use key to decrypt information:

$$
\begin{gathered}
F_{e}=\operatorname{DN}\left(K_{e}\right)=\left(k_{e}-N_{e}\right) \bmod \{92\}, \\
F=F_{0} F_{1} F_{2} \cdots F_{n}, k=k_{0} k_{1} k_{2} \cdots k_{n}, N=N_{0} N_{1} N_{2} \cdots N_{n} .
\end{gathered}
$$

The space-time and space complexity of this algorithm is $u(1)$ and $U(1)$.

By increasing the number of characters, we can reduce the frequency of a single letter. The Vigenère cipher library can be changed from 26 characters to alpha QWERTY password $[8,9]$. It is just that it uses modulus 92 instead of module 26, and ciphertext $\mathrm{C}$ is also extended and harder to crack. After expanding the ciphertext space, the encryption and decryption process is as follows:

Encryption:

$$
Q E\left(F_{1} F_{2} \cdots F_{n}\right)=\left(F_{1}+E_{1}, F_{2}+E_{2}, F_{n}+E_{n}\right)(\bmod 92) .
$$

Decryption:

$$
\operatorname{DN}\left(K_{1} K_{2} K_{3}\right)=\left(K_{1}-N_{1}, K_{2}-N_{2}, K_{n}-N_{n}\right)(\bmod 92) .
$$

In this regard, we improved the above, using LFSR $E_{e+1}$ $=\left(E_{e}+E_{e-1}\right) \bmod \{26\}$ Generate a new key. In addition, this paper also finds a new way to input a short string of characters, and then, the algorithm is obtained:

$$
E_{e}=Q E\left(E_{e}\right)=\left(E_{e-n}+E_{e-n+1}+E_{e-n+2}+\cdots+E_{e-1}\right) \bmod \{26\}(e \geq n),
$$

$$
E_{e}=E_{e}(e<n)
$$

With the continuous improvement of computing power, various original unsolvable mathematical problems have been gradually overcome. Encryption algorithms are usually generated from mathematical problems, so there are no hard-to-understand encryption methods [10, 11]. By trying all possible keys, detailed methods can break any type of password. The disadvantage is that it requires the highest cost. In the Virginia encryption algorithm, the ciphertext attackers crack the Vigenère password. Therefore, the security of the system can be improved by making the frequency of letters in ciphertext evenly distributed. This method uses a new consecutive key $[12,13]$ encrypted from the original key. For example, some scholars propose to add random numbers to the ciphertext, which takes up too much space when many messages need to be transmitted safely. Therefore, we propose a new encryption method combining 
Wigan cipher, LFSR, and OTP, which can make the letters in ciphertext evenly distributed without changing the size of the ciphertext $[14,15]$.

The curve equation of ellipse is as follows:

$$
\begin{gathered}
y^{2}=m_{1} x y+m_{3} y=x^{3}+m_{2} x^{2}+m_{4} x+m_{6}, \\
y^{2}=x^{3}+m x+n(\bmod p) .
\end{gathered}
$$

The following satisfies formula (8):

$$
4 m^{3}+27 n^{2}(\bmod p) \neq 0
$$

Elliptic curve $e$ is composed of infinite point $O$ and point $(x, y)$ satisfying formula (6). It can be proven that the known $K$ and $P$ can easily calculate the value of $Z$, while knowing the value of $Z$ and $P$ to calculate the $K_{16}$ value is very difficult; even for the present, the value of $K$ cannot be obtained. It is also the principle of ECC security $[16,17]$.

2.2. Comparative Analysis. This paper focuses on the application of blockchain technology in the existing crossborder e-commerce payment mode. In the e-commerce payment mode, using blockchain technology is more secure and reliable than the existing supply chain. For enterprises, this mode is implemented efficiently, a decentralized distributed ledger is for the public, and each participant is a node of the blockchain without intermediate transition consumption. This reduces costs [18]. Based on the development of the current e-payment mode, its innovation should first focus on the decentralized distributed theory and establish a distributed ledger $[19,20]$. The widely used third-party payment has gradually developed into a wide range of node-connected networks [21, 22]. In this network, all access ports are open to the public, and all participating connections improve the reputation and transparency. The relationship diagram is shown in Figure 2.

The whole distributed network is development of network. Advanced mathematics and encryption methods include not only the sharing and supervision of transaction content but also the control ability of private information. In general, even in the process of transaction, innovative epayment can save time, space, manpower, and management costs and realize the integration of automation, intelligence, process, and multiple supervision, so as to build a new sharing mode of electronic payment. At present, the electronic sharing mode based on the above analysis needs several characteristics of a core technology to carry out experiments. Through the development of the application of bitcoin technology in the existing electronic payment methods, blockchain technology, as the basis of bitcoin technology, emerges as the times require. The combination of blockchain technology and electronic payment has become a new development opportunity and trend of electronic payment.

2.3. Case Analysis. When introducing the existing supply chain and e-commerce payment system based on blockchain technology, relevant examples are cited. When describing the existing e-commerce payment system, it lists the prod-

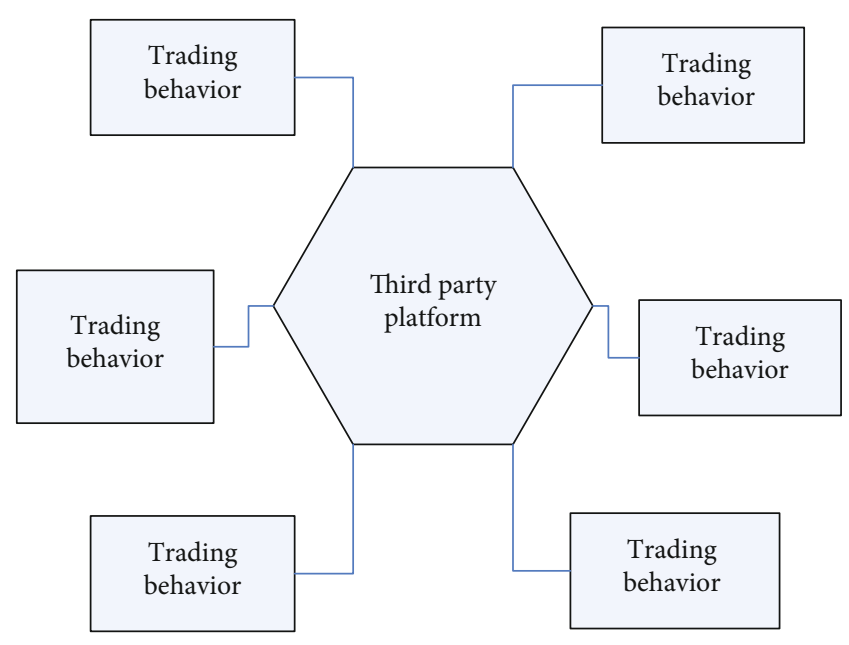

FIgURE 2: E-commerce payment diagram.

ucts of several commercial banks; it also introduces several cases to analyze the current practice of the blockchain platform.

\section{Research Design}

3.1. Design of the Information Encryption and Decryption Algorithm. The security network payment system performance was evaluated. Figure 3 shows the execution process of the mixed encryption program:

(1) A user sends a corresponding private key

(2) The JR server passes the public key to encrypt DES generated by itself

(3) The user passes the encrypted DES key to the server

(4) A secure channel is established in which both the server and the client can interpret each other. The advantage of this is that if missing, it cannot be explained that the DES key is missing. User data cannot be interpreted without the RSA private key required to decrypt. The dual hybrid encryption technology can ensure the security of user data

3.2. Optimization of E-commerce Online Payment Mode. At present, the application of network payment is based on the basic structure and standard of Internet, which involves a wide range of fields from communication protocol to application integration. Therefore, the security measures are mapped to the corresponding levels, which can better describe the online payment security technology system. Generally speaking, the online payment security technology is divided into layers, and the main technical components are shown in Figure 4.

This result is followed by a prefilled message length in 64-bit binary format, because the information length is required in subsequent processing, which will run in four cycles. 


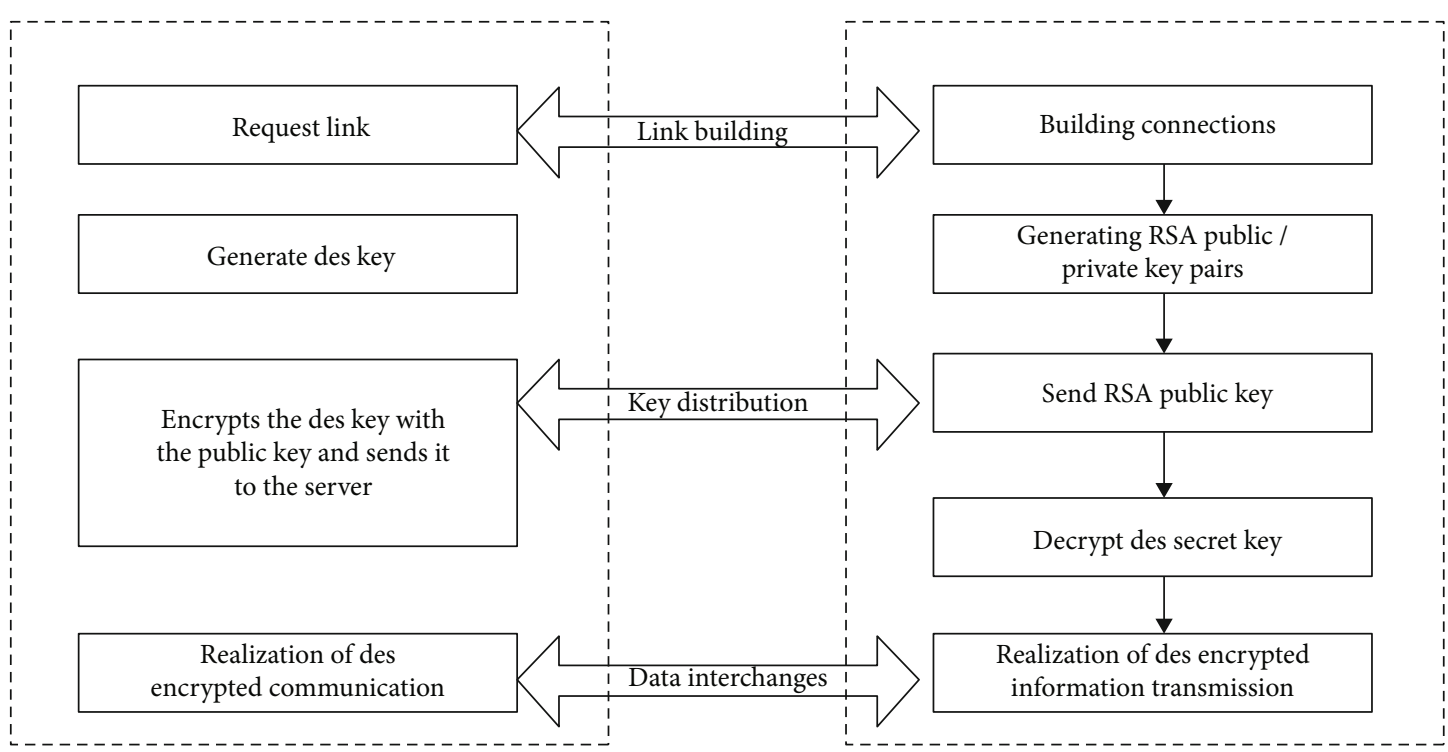

FIGURE 3: Flow chart of information mixed encryption program execution.

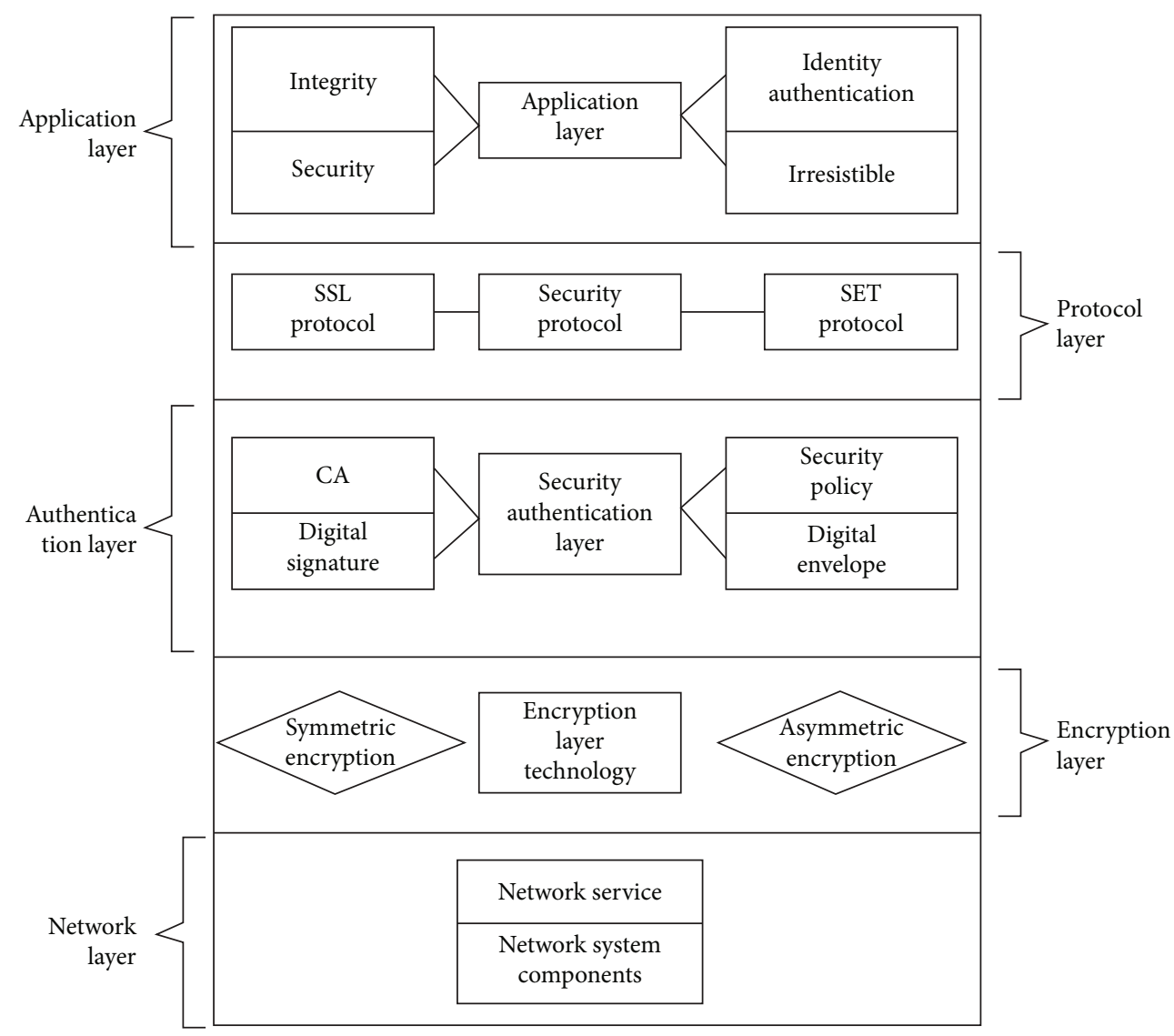

Figure 4: Safety diagram of all levels.

Copy the above four uncertainties into the var4o. The main loop has four rounds, each of which is very similar. Sixteen operations were performed in the first round. Data of the function operation are used to operate on the remain- ing variables, the result of the operation is moved to a random number, and then one of var2, var3, or VAR4 is added to Varl. Finally, replace one of Varl, var2, var3, or VAR4 with this result. 
TABle 1: Paymen flow properties.

\begin{tabular}{|c|c|c|}
\hline Property name & Type & Definition \\
\hline [PaymentFlow_id] & [bigint] & Object unique primary key \\
\hline [GatewayType] & [varchar] $(20)$ & Payment gateway type \\
\hline [GatewayUniqueCode] & {$[\operatorname{varchar}](50)$} & Payment gateway unique code \\
\hline [FlowStatus] & [varchar] $(20)$ & Abstract payment status \\
\hline [FlowToken] & [varchar] $(50)$ & Abstract payment voucher code \\
\hline [RefNumber] & {$[$ varchar] $(50)$} & Payment reference number \\
\hline [Amount] & [decimal] $(18,2)$ & Payment amount \\
\hline
\end{tabular}

Table 2: Properties of the Paymen flow log object.

\begin{tabular}{lcc}
\hline Property name & Type & Definition \\
\hline$[$ PaymentFlowLog_id $]$ & {$[$ bigint $]$} & Object unique primary key \\
{$[$ PaymentFlow_id] } & {$[$ bigint $]$} & Payment flow number, foreign key \\
{$[$ EventType $]$} & {$[$ varchar $](20)$} & Event type \\
{$[$ ProcessMachine $]$} & {$[$ varchar $](50)$} & Processing server name \\
{$[$ Detail $]$} & {$[$ ntext $]$} & Log details \\
{$[$ InsertDate $]$} & {$[$ datetime $]$} & Record creation time \\
{$[$ InsertBy_id $]$} & {$[$ int $]$} & Record creator ID \\
{$[$ ClientAuditingInfo] } & {$[$ nvarchar $](50)$} & Details of audit information \\
\hline
\end{tabular}

$$
\begin{gathered}
f(x, y, z)=(x \vartheta y)(\sim x \partial z), \\
g(x, y, z)=(x \vartheta x)(y \partial z), \\
h(x, y, z)=(x A y A z), \\
i(x, y, z)=y A(x \mid)(\sim z),
\end{gathered}
$$

The four functions were explained: if each bit of the three objects $x, y$, and $Z$ of the function operation is independently and evenly distributed, the result of the function operation will also be independent and uniform. If $f$ is $x$, then $f$ is a bitwise operation; if $x$ is $y$, it is otherwise $Z$. Function $H$ is a bitwise parity operator.

If you enter 123456, el0adc3949ba59abbe56e057f20f883e is encrypted. Therefore, if someone obtains encrypted data, it is difficult to obtain data before encryption.

\section{Analysis of Research Results of Blockchain Finance on Cross-Border E-commerce Payment Security}

4.1. Process Optimization Analysis of the Cross-Border Ecommerce Payment Gateway. For system implementation, this paper first defines the data object and database storage table and implements the data persistence method in the system framework. In addition, the payment gateway downstream of the system defines the abstract protocol interface of the payment gateway. The interface uniformly describes the remote call function to be connected when adding a payment gateway. Through this hierarchical division, the integrated system can be decoupled, updated, debugged, or replaced, which is much easier than the strong coupling mode. It can also effectively reduce file conflicts in the process of code writing by multiple team members. Different payment gateways can be implemented at the same time to improve team efficiency. The payment process object, which represents a single transaction request, includes two types: payment and refund. The payment process is the core object in the payment module, and some of its properties are shown in Table 1.

A payment process object, which is the subordinate object of the payment process, is used to record the step details of interactive processing of the payment method. It is not directly oriented to payment business but mainly provides help for technical personnel in fault diagnosis. At the same time, it also has a certain auxiliary audit function. Its attributes are shown in Table 2.

In the e-commerce payment system, a sequence diagram is one of the dynamic views to describe business scenarios. In this paper, we use a sequence diagram to describe the payment process and make state parameter pattern analysis.

In all online payment businesses, the core content is the payment process, which is directly related to the department and unifies the logical rationality and fluency of business processing. The specific payment process of the payment gateway system is shown in Figure 5.

(1) Users browse the goods on the websites of merchants and enterprises, and then put them into the shopping cart and execute the corresponding unified order procedures. (2) Enterprises fill in and place orders according to the purchase requests sent by customers and send the payment request information to the payment transaction network system. (3) The payment transaction network system checks the effectiveness of merchants and verifies the payment 


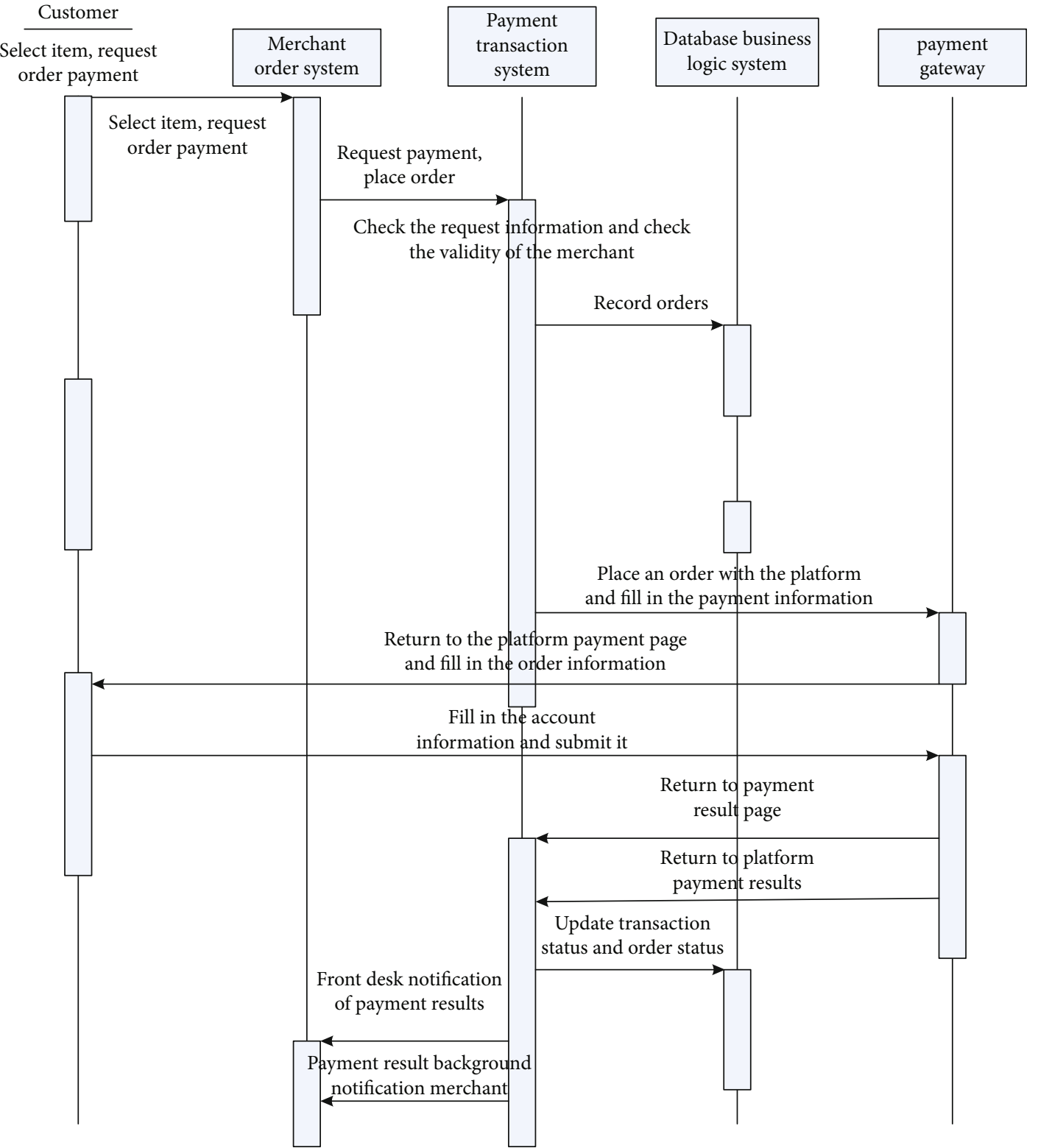

Figure 5: Flow chart of the payment gateway system.

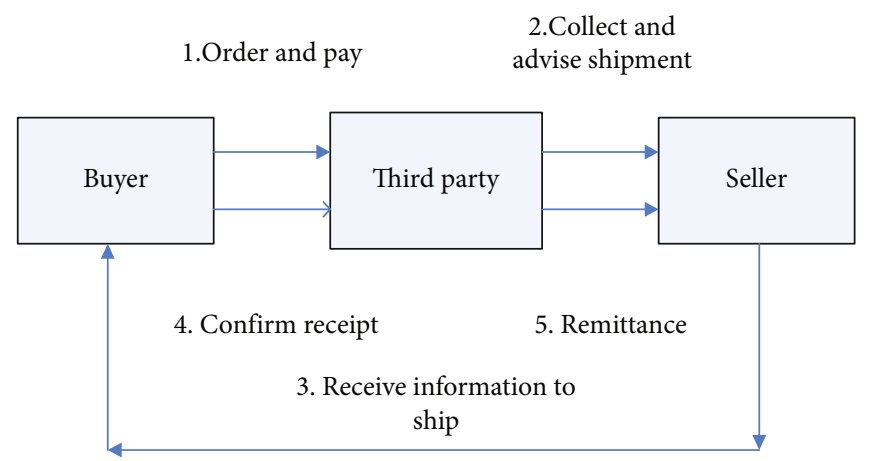

FIgURE 6: Centralized payment process. 


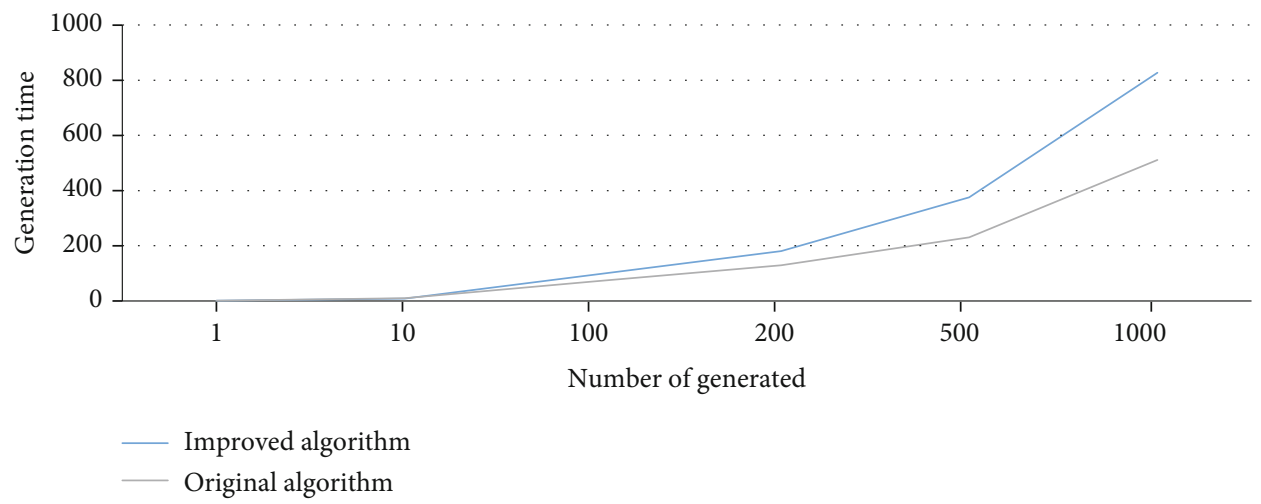

Figure 7: Contrast diagram of scheme generation time.

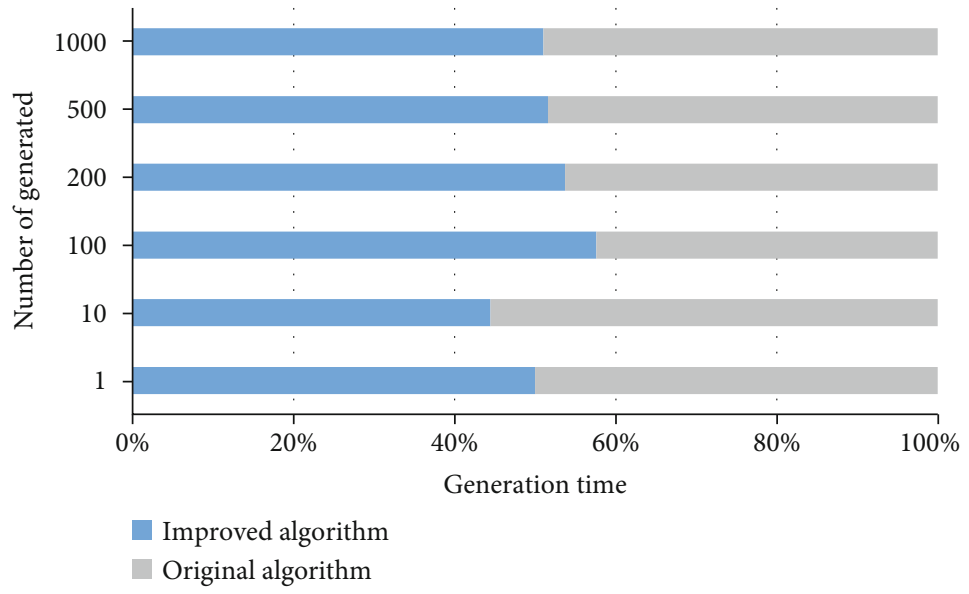

FIGURE 8: Improvement efficiency comparison chart.

request information and then passes through the persistence layer. Store the order information and transaction information in the database. (4) Check whether the merchant request contains the third-party platform channel information; if so, submit the order information to the page in the browser. (5) Provide the third-party platform list and order information to the customer, and the customer can choose the payment institution independently. (6) Submit the payment transaction request and send it to the network system. (7) Update the order information. (8) Submit the updated order information to the corresponding third-party platform, and display the payment page of the third-party platform through the browser. (9) Return to the payment page. (10) Fill in the account information and submit after confirmation. (11) Process the payment information through the background server of the third-party platform and generate the payment result information. The payment transaction network system processes the returned results from the third-party platform, updates the order list, and sends the payment result information to merchants and customers. The technical implementation of the system is a comprehensive process combining macrodesign with specific details, and it can complete the best integration with the payment module. According to the business requirements of the upstream business system, some unnecessary functions can be deleted. Coordinate and optimize the whole system to improve the overall efficiency.

The most important change of electronic payment based on blockchain is the change of the payment process based on tripartite and decentralization. The centralized payment process is shown in Figure 6.

From the above process, we can see that although the whole payment transaction process is between the buyer and the seller, the key steps are completed in the third party. At present, this kind of transaction process is widely used because of the position advantage of the third party; that is to say, no matter whether there are problems in any link of the transaction process, the buyer and the seller can provide solutions through the mutual trust of the third party platform to achieve the ultimate win-win situation.

4.2. Performance Analysis of the Payment Security Algorithm. In order to compare the efficiency of the improved DES and RSA generation algorithm and the original algorithm by using blockchain technology, this paper adopts the method of calling the server side from the Fabric-CA client which, respectively, uses the improved DES and RSA generation algorithm. Each data is the average value of twenty groups 
of data excluding extreme values under the same variable. As shown in Figure 7, the generation time of the original generation scheme and the improved scheme is compared.

As shown in Figure 8, because a very short key can generate a long period, as long as the period length of the new key is larger than the plaintext length, the length of the original key has little effect on the final ciphertext frequency distribution. Therefore, in the period range, the original key with short length can be selected to save the resource consumption during transmission. We further compare the two encryption algorithms through data analysis. Compared with the traditional algorithm, the new algorithm has a smaller letter coincidence index. This paper first summarizes on this basis the process of using the concept of blockchain finance to improve DES and RSA generation algorithm and optimize the security payment process and explains the reason and purpose of this chapter to improve the algorithm. This paper introduces the workflow and improvement direction of the DES and RSA generation algorithm, puts forward specific improvement measures, and explains the effect of improvement through data and chart analysis. It is secure in frequency analysis attacks. At the same time, in our proposed algorithm, the original key with short length can reduce and can increase the key period nonlinearly. In addition, we try to expand the ciphertext space.

The reason why the cross-border e-commerce payment mode with blockchain technology can improve the efficiency is that the distributed data structure ensures that all users in the supply chain have equal rights in information acquisition. Blockchain is similar to a distributed bookkeeping book. Each subject in the chain is at the same level, and the third-party platform of banking is no longer responsible for bridging the lead for the intermediate contact. In the distributed structure, digital signature enables all users to obtain common information, and everyone knows the situation of all traders, which improves the payment efficiency and ensures the security. Banks do not need to rely on the intermediate core enterprises as supervisors to inquire about the actual situation past, which saves time cost and human resources.

\section{Conclusions}

Based on blockchain, this payment method is still in its infancy, and it is also facing technical challenges and traditional concept challenges. However, the progress of blockchain technology application itself is relatively slow, and the application of cross-border electronic payment needs to be improved and developed. There is still a gap in technical factors. There are still many aspects to be improved and discussed, including some other factors that may have an important impact on it, such as network delay in the process of encryption and authentication, information transmission, and storage problems between modules, and block capacity is also an uncertain factor. In order to achieve efficient transaction and payment, we need to further improve the information system to achieve high-level requirements.

Starting from the concept of e-commerce and payment platform-related business, this paper combs and analyzes the development of payment platform business domestically and internationally. On this basis, it improves the payment security algorithm of the e-commerce payment platform from various aspects, optimizes the traditional payment process, and discusses several typical payment models. On this basis, the concept of blockchain finance is introduced and applied to the security optimization of payment mode. The payment security algorithm and payment process are improved and optimized.

Generally speaking, international exchanges are constantly strengthening, international trade is growing, and cross-border e-commerce is developing rapidly. However, in today's changing social and economic status, not only is cross-border e-commerce always facing opportunities and challenges but also cross-border e-payment mode needs to be constantly innovated with the changes of the times. Blockchain technology has brought new development opportunities for cross-border electronic payment mode, which is not only the progress of technology but also the needs of the times.

\section{Data Availability}

The data that support the findings of this study are available from the corresponding author upon reasonable request.

\section{Conflicts of Interest}

The authors declared no potential conflicts of interest with respect to the research, authorship, and/or publication of this article.

\section{References}

[1] I. Eyal, "Blockchain technology: transforming libertarian cryptocurrency dreams to finance and banking realities," Computer, vol. 50, no. 9, pp. 38-49, 2017.

[2] D. Puthal, N. Malik, S. P. Mohanty, E. Kougianos, and G. Das, "Everything you wanted to know about the blockchain: its promise, components, processes, and problems," IEEE Consumer Electronics Magazine, vol. 7, no. 4, pp. 6-14, 2018.

[3] L. Chen and R. Wang, "Trust development and transfer from electronic commerce to social commerce: an empirical investigation," American Journal of Industrial and Business Management, vol. 6, no. 5, pp. 568-576, 2016.

[4] C. W. Holsapple and M. Singh, "Toward a unified view of electronic commerce, electronic business, and collaborative commerce: a knowledge management approach," Knowledge \& Process Management, vol. 7, no. 3, pp. 151-164, 2000.

[5] N. Radziwill, "Blockchain revolution: how the technology behind bitcoin is changing money, business, and the world," Quality Management Journal, vol. 25, no. 1, pp. 64-65, 2018.

[6] M. Iansiti and K. R. Lakhani, "The truth about blockchain," Harvard Business Review, vol. 95, no. 1, pp. 118-127, 2017.

[7] D. Yermack, "Corporate governance and blockchains," Review of Finance, vol. 21, no. 1, pp. 7-31, 2015.

[8] J. Kang, R. Yu, X. Huang, S. Maharjan, Y. Zhang, and E. Hossain, "Enabling localized peer-to-peer electricity trading among plug-in hybrid electric vehicles using consortium 
blockchains," IEEE Transactions on Industrial Informatics, vol. 13, no. 6, pp. 3154-3164, 2017.

[9] A. Bahga and V. K. Madisetti, "Blockchain platform for industrial Internet of things," Journal of Software Engineering and Applications, vol. 9, no. 10, pp. 533-546, 2016.

[10] J. Göbel, H. P. Keeler, A. E. Krzesinski, and P. G. Taylor, "Bitcoin blockchain dynamics: the selfish-mine strategy in the presence of propagation delay," Performance Evaluation, vol. 104, pp. 23-41, 2016.

[11] A. Ouaddah, A. Abou Elkalam, and A. Ait Ouahman, "FairAccess: a new blockchain-based access control framework for the Internet of things," Security and Communication Networks, vol. 9, no. 18, 5964 pages, 2016.

[12] M. A. Khan and K. Salah, "IoT security: review, blockchain solutions, and open challenges," Future Generation Computer Systems, vol. 82, pp. 395-411, 2017.

[13] Y. Guo and C. Liang, "Blockchain application and outlook in the banking industry," Financial Innovation, vol. 2, no. 1, 2016.

[14] A. Dorri, M. Steger, S. S. Kanhere, and R. Jurdak, "BlockChain: a distributed solution to automotive security and privacy," IEEE Communications Magazine, vol. 55, no. 12, pp. 119125, 2017.

[15] T. T. A. Dinh, R. Liu, M. Zhang, G. Chen, B. C. Ooi, and J. Wang, "Untangling blockchain: a data processing view of blockchain systems," IEEE Transactions on Knowledge \& Data Engineering, vol. 99, 2017.

[16] E. Mengelkamp, B. Notheisen, C. Beer, D. Dauer, and C. Weinhardt, "A blockchain-based smart grid: towards sustainable local energy markets," Computer Science-Research and Development, vol. 33, no. 1-2, pp. 207-214, 2018.

[17] H. Zhao, M. Zhang, S. Wang, E. Li, Z. Guo, and D. Sun, "Security risk and response analysis of typical application architecture of information and communication blockchain," Neural Computing and Applications, vol. 33, no. 13, pp. 7661-7671, 2021.

[18] C. Pop, T. Cioara, M. Antal, I. Anghel, I. Salomie, and M. Bertoncini, "Blockchain based decentralized management of demand response programs in smart energy grids," Sensors, vol. 18, no. 2, p. 162, 2018.

[19] L. Deng, D. Li, Z. Cai, and X. Yao, “A QoS optimization system for complex data cross-domain request based on neural blockchain structure," Neural Computing and Applications, vol. 32, no. 21, pp. 16455-16469, 2020.

[20] Q. Xia, E. Sifah, A. Smahi, S. Amofa, and X. Zhang, "BBDS: blockchain-based data sharing for electronic medical records in cloud environments," Information, vol. 8, no. 2, p. 44, 2017.

[21] D. Tapscott and A. Tapscott, "How blockchain will change organizations," MIT Sloan Management Review, vol. 58, no. 2, pp. 10-13, 2017.

[22] P. K. Sharma, S. Y. Moon, and J. H. Park, "Block-VN: a distributed blockchain based vehicular network architecture in smart city," Journal of Information Processing Systems, vol. 13, no. 1, pp. 184-195, 2017. 